\title{
Ab initio molecular dynamics study of liquid methanol
}

\author{
Jan-Willem Handgraaf, Titus S. van Erp, and Evert Jan Meijer \\ Department of Chemical Engineering, University of Amsterdam, Nieuwe \\ Achtergracht 166, 1018 WV AMSTERDAM, The Netherlands \\ Fax:+31-20-5255604, email: ejmeijer@science.uva.nl
}

\begin{abstract}
We present a density-functional theory based molecular-dynamics study of the structural, dynamical, and electronic properties of liquid methanol under ambient conditions. The calculated radial distribution functions involving the oxygen and hydroxyl hydrogen show a pronounced hydrogen bonding and compare well with recent neutron diffraction data, except for an underestimate of the oxygen-oxygen correlation. We observe that, in line with infrared spectroscopic data, the hydroxyl stretching mode is significantly red-shifted in the liquid. A substantial enhancement of the dipole moment is accompanied by significant fluctuations due to thermal motion. Our results provide valuable data for improvement of empirical potentials.
\end{abstract}

\section{Introduction}

Liquid methanol is of fundamental interest in natural sciences and of significant importance in technical and industrial applications. The liquid phase of the simplest alcohol is widely studied, both experimentally and theoretically. Among the alcohols, methanol is the closest analog to water. The characteristic hydroxyl group allows methanol to form hydrogen bonds that dominate the structural and dynamical behavior of the liquid phase. The methyl group does not participate in the hydrogen bonding and constitutes the distinction with water. This difference is apparent in the microscopic structure of the liquid, with water having a tetrahedral-like coordination, whereas for methanol experiments and molecular simulation suggest a local structure consisting of chains, rings, or small clusters.

The precise quantification of the microscopic structural and dynamical picture of liquid methanol has been a long-time subject in both experimental 
and molecular simulation studies. Recently, a series of state-of-the-art studies have been reported. Among these are the neutron diffraction (ND) experiments of Refs. [1,2]. Simulation studies include work based on empirical force-fields[3,4], mixed empirical and ab-initio interactions[5,6], and a full ab initio molecular dynamics study[7]. The recent ND experiments have provided a detailed microscopic picture of the structure of liquid methanol, including the pair distribution functions among all atoms. Yet, some of these atomatom distribution functions are still subject to some uncertainty as they are obtained indirectly.

Molecular simulation provides a complementary approach to study the microscopic behavior of liquids. Most molecular simulations studies of liquid methanol are based on empirical force fields potentials that are designed to reproduce a selection of experimental data. Obviously, molecular simulations based on these potentials do not provide a picture completely independent from experiment. Moreover, the reliability of the results at conditions that are significantly different from those where the potential was designed for, may be questionable. Density functional theory (DFT) based molecular dynamics (MD) simulation, such as the Car-Parrinello molecular dynamics method[8], where the interactions are calculated by accurate electronic structure calculations provides a route to overcome these limitations. This has been demonstrated in studies of liquid water $[9,10,11]$ and aqueous solvation $[12,13,14]$. Important advantages of DFT-MD over force-field MD are that it intrinsically incorporates polarization, that it accounts for the intra-molecular motion and therefore allows for a direct comparison with spectroscopy of intra-molecular vibrations, and that it yields detailed information on the electronic properties, such as the energy levels of electronic states and the charge distribution. In a broader chemical perspective it is important to note that DFT-MD is capable of the study of chemical reactions in solution, where force-field MD would fail completely as it cannot account for the change in chemical bonding.

Here, we report a DFT-MD study of liquid methanol that addresses the liquid structure, the inter- and intra-molecular dynamics, and the electronic charge distribution.

\section{Methods and Validation}

Electronic structure calculations are performed using the Kohn-Sham formulation of DFT. We employed the gradient-corrected BLYP functional[15,16]. The choice for the BLYP functional was guided by its good description of the structure and dynamics of water[10] where hydrogen bonds are, as in liquid methanol, the dominant interactions. Furthermore, it has been shown that DFT-BLYP gives a proper description of solvation of methanol in water[13]. 
The DFT-based MD simulations are performed with the Car-Parrinello method $[8,17]$ using the CPMD package[18]. Semi-local norm-conserving Martins-Troullier pseudopotentials[19] are used to restrict the number of electronic states to those of the valence electrons. The pseudopotential cut-off radii were taken $0.50,1.11$ and 1.23 a.u, for $\mathrm{H}, \mathrm{O}$, and $\mathrm{C}$, respectively. The electronic states are expanded in a plane-wave basis with a cut-off of 70 Ry yielding energies and geometries converged within $0.01 \AA$ and $1 \mathrm{~kJ} / \mathrm{mol}$, respectively. Vibrational frequencies are converged within $1 \%$, except for $\mathrm{C}-\mathrm{O}$ and $\mathrm{O}-\mathrm{H}$ stretch modes that are underestimated by $3 \%$ and $5 \%$ compared to the basis-set limit values[13].

To validate our computational approach we compared results for the gas-phase monomer and hydrogen-bonded dimer against state-of-the-art atomic-orbital DFT calculations obtained with ADF[20], $\square$ and against the B3LYP and MP2 calculations of Ref. [21]. The CPMD-BLYP calculations were performed using a cubic box with an edge of $12.9 \AA$, with the interactions among the periodic images eliminated by a screening technique[17]. Results for geometry and complexation energy of the dimer are given in Fig. 1 and Table 1. Deviations among CPMD and ADF are $1 \mathrm{~kJ} / \mathrm{mol}$ for the complexation energy, smaller than $0.01 \AA$ for the intra-molecular bonds, and smaller than $0.02 \AA$ for the hydrogen bond. This indicates a state-of-the-art accuracy for the numerical methods employed in CPMD. Compared to the MP2 and B3LYP results, the BLYP bond lengths are slightly longer, with deviations up to 0.03 and $0.05 \AA$ for the intra- and inter-molecular bond lengths, respectively. Differences among BLYP, B3LYP and MP2 complexation energies are within acceptable limits, with the BLYP energies smaller by $2-4 \mathrm{~kJ} /$ mole. The deviations are similar to the comparison between BLYP[10] and MP2 $2^{2}$ for the water dimer and the water-methanol dimer[23,13]. We obtained a zero-Kelvin association enthalpy $\Delta H^{0}(0)$ of $10.6 \mathrm{~kJ} / \mathrm{mol}$ using the B3LYP zero-point energy of Ref. [21]. This is in reasonable agreement with the experimental value of $13.2(4) \mathrm{kJ} / \mathrm{mol}$. The calculated hydrogen bond length $\left(r_{\mathrm{O}} \ldots \mathrm{O}=2.94 \AA, r_{\mathrm{H}} \ldots \mathrm{O}=1.95 \AA\right)$ is in good agreement with the experimental values of $r_{\mathrm{O}} \ldots \mathrm{O}=2.98(2) \AA$ and $r_{\mathrm{H} . . \mathrm{O}}=1.96(2) \AA$ of Refs. [24] and [25], respectively.

Current gradient corrected functionals such as BLYP do not account for dispersion forces. For methanol this could be important as attraction to the methyl group is fully due to the dispersion force. To estimate the effect of the absence of the dispersion we computed the BLYP binding energy of two dimer configurations that are sensitive to this: one with methyl groups approaching (M-M) and the other with the methyl and hydroxyl group approaching each

$\overline{1}$ Kohn-Sham orbitals are expanded in an even-tempered, all-electron Slater type basis set augmented with $2 \mathrm{p}$ and $3 \mathrm{~d}$ polarization functions for $\mathrm{H}$ and $3 \mathrm{~d}$ and $4 \mathrm{f}$ polarization functions for $\mathrm{C}$ and $\mathrm{O}$.

2 MP2 limit estimate. See for example [22] 
other (M-OH). State-of-the art MP2 calculations[26], that incorporate to a good approximation the dispersion force, serves as a reference. The dimer configurations were taken from Ref. [26] and chosen such that the carboncarbon (M-M dimer) and carbon-oxygen (M-OH dimer) distances were close to the peak position of their atom-atom distribution function in the liquid state. ${ }^{3}$ The comparison yields for the M-M dimer values of $2.3 \mathrm{~kJ} / \mathrm{mol}$ and $-2.0 \mathrm{~kJ} / \mathrm{mol}$ for BLYP and MP2, respectively. For the $\mathrm{M}-\mathrm{OH}$ dimer these values are $-1.2 \mathrm{~kJ} / \mathrm{mol}$ and $-4.9 \mathrm{~kJ} / \mathrm{mol}$, respectively. The too repulsive nature of the BLYP interaction is consistent with DFT calculations of dispersion dominated systems $[27,28]$. However, although by far not insignificant, the magnitude of the deviation is much smaller than the hydrogen-bond interaction and of the same order of magnitude as the error in the latter. It can therefore be argued that for a study of liquid methanol on the accuracy level of BLYP, neglecting the dispersion interaction is acceptable.

In Ref. [13] we have shown that for the gas-phase monomer CPMD-BLYP vibrational frequencies are in excellent agreement with ADF results and, compared to experiment, underestimate allmost all modes by $\approx 10 \%$, a known feature of the BLYP functional.

Overall, we conclude that our level theory is satisfactory in comparison with experimental and other theoretical gas-phase data.

\section{Liquid}

Liquid methanol was modeled by 32 molecules in a periodic cubic box with an edge of $12.9 \AA$, reproducing the experimental density of $0.791 \mathrm{~g} / \mathrm{cm}^{3}$ at 293 K[29]. The temperature was fixed at $293 \mathrm{~K}$ using the Nosé-Hoover thermostat [30]. The fictitious mass associated with the plane-wave coefficients is chosen at 900 a.u., which allowed for a time step in the numerical integration of the equations-of-motion of 0.145 fs. The system was equilibrated for 1 ps from an initial configuration obtained from a force-field simulation. Subsequently, we gathered statistical averages from a 6 ps trajectory.

\subsection{Structure}

In Fig. 2 we have plotted the most characteristic atom-atom radial distribution functions (RDFs), i.e. the hydrogen bonding $\mathrm{O}-\mathrm{O}, \mathrm{O}-\mathrm{H}_{\mathrm{O}}$, and $\mathrm{H}_{\mathrm{O}}-\mathrm{H}_{\mathrm{O}} \mathrm{RDF}$ and

3 Configuration from Ref. [26]. M-M dimer: geometry $\mathrm{M}$ with $r_{\mathrm{CC}}=3.75 \AA$. M-OH dimer: geometry I with $r_{\mathrm{CO}}=3.50 \AA$. Both monomers in the dimer are kept fixed to their isolated geometries. 
the C-O RDF. For comparison we also plotted results of recent ND results[1] and the peak positions obtained using Haugney's empirical potential[3], the latter being considered one of the most accurate empirical force fields to date.

The pronounced structure in the first three RDFs are a clear indication of the presence of hydrogen bonds. Comparison with the experimental data shows that the positions of the first peaks match within the statistical error for the $\mathrm{O}-\mathrm{O}$ and $\mathrm{O}-\mathrm{H}_{\mathrm{O}}$ RDFs and is slightly smaller $(\approx 0.1 \AA)$ for the $\mathrm{H}_{\mathrm{O}}-\mathrm{H}_{\mathrm{O}} \mathrm{RDF}$. The height of the first peak is in good agreement for the $\mathrm{O}-\mathrm{H}_{\mathrm{O}}$ and $\mathrm{H}_{\mathrm{O}}-\mathrm{H}_{\mathrm{O}}$ RDFs, that both can be determined accurately from the ND data. However, the O-O RDF shows a calculated first peak height that is significantly lower than the experimental result. Given the small system of 32 molecules in our simulation, the discrepancy could well be a system-size effect. On the other hand, the indirect way by which the the O-O RDF is extracted from ND data could yield an overestimate of the $\mathrm{O}-\mathrm{O}$ correlation. Comparison with force field results[3], that yield significant higher peak values for the $\mathrm{O}-\mathrm{O}$ and $\mathrm{O}-$ $\mathrm{H}_{\mathrm{O}}$ RDFs, suggests that the Haugney potential overestimates the hydrogen bonding structure in the liquid, in line with the observation of Ref. [1].

The number of $\mathrm{H}$-bonds as calculated by integrating the $\mathrm{O}-\mathrm{H}_{\mathrm{O}}$ and $\mathrm{O}-\mathrm{O}$ RDFs up to the first minimum, and using the geometrical criterion of Ref. [3], yields values of 1.9, 2.0, and 1.6, respectively. This is in good agreement with the experimental ND results of Ref. [1] yielding 1.8 and 1.9 obtained by integrating the $\mathrm{O}-\mathrm{H}_{\mathrm{O}}$ and O-O RDFs. Applying the geometrical criterion to the Haugney force-field simulation[3] yields a slightly higher value of 1.9.

The hydrogen bonding in the liquid phase is accompanied by an elongation of the $\mathrm{OH}_{\mathrm{O}}$ bond of $0.15 \AA$. A direct comparison with the experimental results for this change in the geometry of the methanol molecule is rather difficult due to the large spread in the reported values. However, similar change in the geometry is observed in the DFT-MD study of liquid methanol reported in Ref. [7] and in ab initio studies of small methanol clusters[21].

The calculated $\mathrm{C}-\mathrm{O} \mathrm{RDF}$ is in reasonable agreement with the ND results, with the overall shape well reproduced but the first peak clearly less pronounced than in the ND result. This is consistent with the, in the previous section found, underestimation of the BLYP binding energy of the M-O dimer with a C-O distance at the RDF peak position. This is due to the absence of the dispersion interaction in BLYP. However, the absence of the dispersion interaction clearly does not lead to a completely distorted C-O positional correlation. The comparison of the calculated $\mathrm{C}-\mathrm{C}$ RDF with the ND result (not plotted) is very similar. 


\subsection{Dynamics}

The time scale of the present simulation (6 ps) allows for an analysis of the short-time dynamics of liquid methanol. Figure 3 shows the power spectrum of the velocity auto correlation function (VACF) of the hydroxyl hydrogen. For comparison we have also plotted the calculated $200 \mathrm{~K}$ monomer spectrum of Ref. [13]. The three distinct peaks correspond to the $\mathrm{OH}$ stretch $\left(3100 \mathrm{~cm}^{-1}\right)$, $\mathrm{C}-\mathrm{O}-\mathrm{H}$ bend $\left(1600 \mathrm{~cm}^{-1}\right)$, and the CO stretch $\left(1000 \mathrm{~cm}^{-1}\right)$. The broad feature below $1000 \mathrm{~cm}^{-1}$ indicates the librational-translational $\left(500 \mathrm{~cm}^{-1}\right)$ modes of the methanol molecules. Compared to the gas phase, the liquid $\mathrm{OH}$ stretch mode has red-shifted by approximately $200 \mathrm{~cm}^{-1}$ and broadened considerably. On the other hand, the C-O-H bending is blue-shifted by approximately $70 \mathrm{~cm}^{-1}$. The observed shifts and broadening are characteristic for hydrogen bonded liquids and also observed in the spectrum of water or hydrated methanol. The calculated shifts compare reasonably well with experimental infrared spectra[31] that yield values of $-354 \mathrm{~cm}^{-1}$ and $+78 \mathrm{~cm}^{-1}$ for the O$\mathrm{H}$ stretch and $\mathrm{C}-\mathrm{O}-\mathrm{H}$ bend. The calculated positions and shifts of the modes match within statistical errors with those of methanol in aqueous solution[13] determined using the same computational approach. This indicates that the intra-molecular dynamics of methanol is affected in a similar way by an aqueous environment and a methanol environment.

The diffusion constant $D$ is a key measure of the collective dynamics. In view of the limited length of the calculated trajectory we can only provide a rough estimate. From the mean square displacement of the oxygen atoms we obtained $D=2.0 \pm 0.6 \times 10^{-9} \mathrm{~m}^{2} / \mathrm{s}$, in reasonable agreement with the experimental value of $2.42 \pm 0.05 \times 10^{-9} \mathrm{~m}^{2} / \mathrm{s}[32]$.

\subsection{Electronic properties}

As the electronic structure is an intrinsic part of a CPMD simulation, detailed information on the electronic charge distribution is obtained. To quantify the charge distribution we used the method of maximally localized Wannier functions that transforms the Kohn-Sham orbitals into Wannier functions whose centers (WFC) can be assigned with a chemical meaning such as being associated with an electron bonding- or lone-pair (LP)[33].

We calculated the positions of the WFCs for the monomer, the dimer, and 6 independent configurations of the liquid simulation. Table 2 lists the (average) distances of the WFCs associated with the oxygen electrons. Most notably is the small but significant shift of $0.024 \AA$ for the $\mathrm{OH}$ bond WFC towards the oxygen atom when going from the monomer to the liquid. At the same time, 
one of the LP WFCs shifts away from the oxygen by $0.023 \AA$. These changes should be considered a manifestation of the hydrogen bonding and the induced polarization among the dipolar methanol molecules in the liquid state.

To quantify the change in the charge distribution in a single number we calculated the molecular dipole moment assuming the electronic charge to be distributed as point charges located on the WFCs. For liquid water it has been shown that such a partitioning of the charges over the molecules yields a unique assignment of the WFCs over distinct molecules[11]. From Table 3, that lists the values for the monomer, dimer, and liquid, we observe a significant enhancement of the dipole moment going from the monomer via the dimer to the liquid. A comparable liquid-state value of $2.39 \mathrm{D}$ has been observed in a coupled empirical and ab initio MP2 study[5]. Note that the value of the dipole moment is somewhat larger than in the Haugney[3] (2.33 D) or AMBER[34] (2.2 D) force field. A second important feature of the electronic charge distribution in the liquid is its fluctuating character due to the thermally driven configurational changes. In Fig. 4 we have plotted the calculated distribution of the dipole moments in the liquid phase. It shows that there is a significant variation ranging from $1.7 \mathrm{D}$ to $3.5 \mathrm{D}$.

\section{Conclusions}

We have demonstrated that $a b$ initio MD is a valuable approach to study the structural, dynamical, and electronic properties of liquid methanol. The calculated pair distribution functions involving the hydroxyl hydrogens correlate well with recent state-of-the-art neutron diffraction experiments of Soper and co-workers. It confirms their finding that one of the benchmark empirical potentials overestimates the hydrogen bonding structure. The calculated oxygen-oxygen radial distribution function shows significantly less structure than the experimental neutron diffraction results. Currently we are studying a larger simulation sample to see whether this is due to the small system size in the present calculation. It could also be a result from an inaccuracy in the experimental result that is obtained in an indirect way. Results for the dimer binding energies and the oxygen-carbon RDF suggest that the absence of the dispersion interaction is notable but has no major impact. Comparing the vibrational spectra of the liquid phase against that of the gas phase monomer shows a significant red shift of the $\mathrm{O}-\mathrm{H}$ stretch accompanied by a smaller blue shift of the $\mathrm{C}-\mathrm{O}-\mathrm{H}$ bend mode, in reasonable agreement with experimental observations with the $\mathrm{O}-\mathrm{H}$ shift somewhat underestimated in our calculation. We quantified the electronic charge distribution using a Wannier function decomposition. A small but measurable shift of the positions of the Wannier function centers when going from the gas-phase to the liquid is accompanied

by a substantial enhancement of the dipole moment. Moreover we have found 
that in the liquid the dipole moment fluctuates significantly with variations up to half the average magnitude. The latter suggest that the assumption made in empirical potentials using a fixed dipole moment is a strong simplification. The present results may be considered valuable data for improvement of empirical potentials for the study of liquid methanol.

\section{Acknowledgements}

We are grateful to A.K. Soper for providing us data of the RDFs of Ref. [1]. J.-W.H. and T.S.v.E acknowledges NWO-CW (Nederlandse Organisatie voor Wetenschappelijk Onderzoek, Chemische Wetenschappen), J.-W.H through PIONIER. E.J.M. acknowledges the Royal Netherlands Academy of Art and

Sciences for financial support. We acknowledge support from the Stichting Nationale Computerfacileiten (NCF) and the Nederlandse Organisatie voor Wetenschappelijk Onderzoek (NWO) for the use of supercomputer facilities.

\section{References}

[1] T. Yamaguchi, K. Hidaka, A. K. Soper, Mol. Phys. 96 (1999) 1159; Erratum, Mol. Phys. 97 (1999) 603.

[2] A. K. Adya, L. Bianchi, C. J. Wormald, J. Chem. Phys. 112 (2000) 4231.

[3] M. Haughney, M. Ferrario, I. R. McDonald, J. Phys. Chem. 91 (1987) 4934.

[4] L. Bianchi, O. N. Kalugin, A. K. Adya, C. J. Wormald, Mol. Simulation 25 (2000) 321.

[5] Y. Tu, A. Laaksonen, Phys. Rev. E 64 (2001) 026703.

[6] M. E. Martín, M. L. Sánchez, F. J. O. del Valle, M. A. Aguilar, J. Chem. Phys. 116 (2002) 1613.

[7] E. Tsuchida, Y. Kanada, M. Tsukada, Chem. Phys. Lett. 311 (1999) 236.

[8] R. Car, M. Parrinello, Phys. Rev. Lett. 55 (1985) 2471.

[9] K. Laasonen, M. Sprik, M. Parrinello, R. Car, J. Chem. Phys. 99 (1993) 9080.

[10] M. Sprik, J. Hutter, M. Parrinello, J. Chem. Phys. 105 (1996) 1142.

[11] P. L. Silvestrelli, M. Parrinello, J. Chem. Phys. 111 (1999) 3572.

[12] D. Marx, M. Sprik, M. Parrinello, Chem. Phys. Lett. 273 (1997) 360.

[13] T. S. van Erp, E. J. Meijer, Chem. Phys. Lett. 333 (2001) 290. 
[14] S. Raugei, M. L. Klein, J. Chem. Phys. 116 (2002) 196.

[15] C. Lee, W. Yang, R. G. Parr, Phys. Rev. B 37 (1988) 785.

[16] A. D. Becke, Phys. Rev. A 38 (1988) 3098.

[17] D. Marx, J. Hutter, Ab initio molecular dynamics: Theory and implementation, in: J. Grotendorst (Ed.), Modern Methods in Algorithms of Quantum Chemistry, Vol. 1 of NIC Series, John von Neumann Insitute for Computing, Jülich, pp. 301-449.

[18] CPMD, version 3.3, developed by J. Hutter, A. Alavi, T. Deutsch, M. Bernasconi, St. Goedecker, D. Marx, M. Tuckerman, and M. Parrinello, MPI für Festkörperforschung and IBM Zurich Research Laboratory (1995-1999).

[19] N. Troullier, J. L. Martins, Phys. Rev. B 43 (1991) 1993.

[20] ADF 2000, G. te Velde, E. J. Baerends et al. Theoretical Chemistry, Vrije Universiteit, Amsterdam.

[21] O. Mó, M. Yáñez, J. Elguero, J. Chem. Phys. 107 (1997) 3592.

[22] M. Schütz, S. Brdarski, P.-O. Widmark, R. Lindh, G. Karlström, J. Chem. Phys. 107 (1997) 4597.

[23] L. González, O. Mó, M. Yáñez, J. Chem. Phys. 109 (1998) 139.

[24] F. J. Lovas, S. P. Belov, M. Y. Tretyakov, W. Stahl, R. D. Suenram, J. Mol. Spectrosc. 170 (1995) 478.

[25] F. J. Lovas, H. Hartwig, J. Mol. Spectrosc. 185 (1997) 98.

[26] W. T. M. Mooij, F. B. van Duijneveldt, J. G. C. M. van Duijneveldt-van de Rijdt, B. P. van Eijck, J. Phys. Chem. A 103 (1999) 9872.

[27] S. Kristyán, P. Pulay, Chem. Phys. Lett. 229 (1994) 175.

[28] E. J. Meijer, M. Sprik, J. Chem. Phys. 105 (1996) 8684.

[29] J. A. Riddick, W. B. Bunger, T. K. Sakano, Organic solvents: physical properties and methods of purification, Wiley, New York, 1986.

[30] S. Nosé, J. Chem. Phys. 81 (1984) 511.

[31] T. Shimanouchi, Tables of molecular vibrational frequencies consolidated, Volume I, National Bureau of Standards (1972).

[32] R. L. Hurle, L. A. Woolf, Aust. J. Chem. 33 (1980) 1947.

[33] P. L. Silvestrelli, N. Marzari, D. Vanderbilt, M. Parrinello, Solid State Commun. 107 (1998) 7.

[34] W. D. Cornell, P. Cieplak, C. I. Bayly, I. R. Gould, K. M. Merz., Jr., D. M. Ferguson, D. C. Spellmeyer, T. Fox, J. W. Caldwell, P. A. Kollmann, J. Am. Chem. Soc. 117 (1995) 5179.

[35] E. V. Ivash, D. M. Dennison, J. Chem. Phys. 21 (1953) 1804. 
Table 1

Complexation energies $(\mathrm{kJ} / \mathrm{mol})$ of methanol dimer shown in Fig. 1. Numbers are bare values without zero-point energy corrections and without entropy contributions.

\begin{tabular}{l|ccc} 
CPMD-BLYP & ADF-BLYP $^{a}$ & B3LYP $^{b}$ & MP2 $^{c}$ \\
\hline 16.4 & 17.3 & 20.6 & 18.4 \\
\hline$a$
\end{tabular}

${ }^{a}$ Refs. [20]

${ }^{b}$ B3LYP/6-311+G(3df,2p) method. B3LYP/6-311+G(d,p) optimized geometries. From Ref. [23].

c G2(MP2) method. MP2(full)/6-311+G(d,p) optimized geometries. ¿From Ref. [23].

Table 2

Electronic charge distribution in terms of Wannier function centers. $d(\mathrm{LP})$ denotes the average distances between a lone pair WFC and the $\mathrm{O}$ nucleus. $d(\mathrm{OH})$ and $d(\mathrm{OC})$ denote the (average) distances between the covalent WFC along the $\mathrm{O}-\mathrm{H}$ bond and the $\mathrm{O}-\mathrm{C}$ bond with the $\mathrm{O}$ nucleus, respectively. All distances are given in $\AA$. Statistical errors for the liquid data are around 0.002 .

\begin{tabular}{lllll}
\hline \hline & $d(\mathrm{LP})$ & $d(\mathrm{LP})$ & $d(\mathrm{OH})$ & $d(\mathrm{OC})$ \\
\hline Monomer & 0.305 & 0.305 & 0.533 & 0.562 \\
Dimer & 0.316 & 0.306 & 0.522 & 0.561 \\
Liquid & 0.328 & 0.309 & 0.509 & 0.561 \\
\hline \hline
\end{tabular}

Table 3

Dipole moment. Experimental value is given in parentheses. Data for the liquid phase were obtained by averaging over 6 configurations of the MD simulation. Statistical errors are in the order of some units in the last digit.

\begin{tabular}{ll}
\hline \hline & $\mu(\mathrm{D})$ \\
\hline Monomer & $1.73\left(1.69^{a}\right)$ \\
Dimer & 2.03 \\
Liquid & 2.54 \\
\hline \hline
\end{tabular}

${ }^{a}$ Microwave study, ref. [35]. 


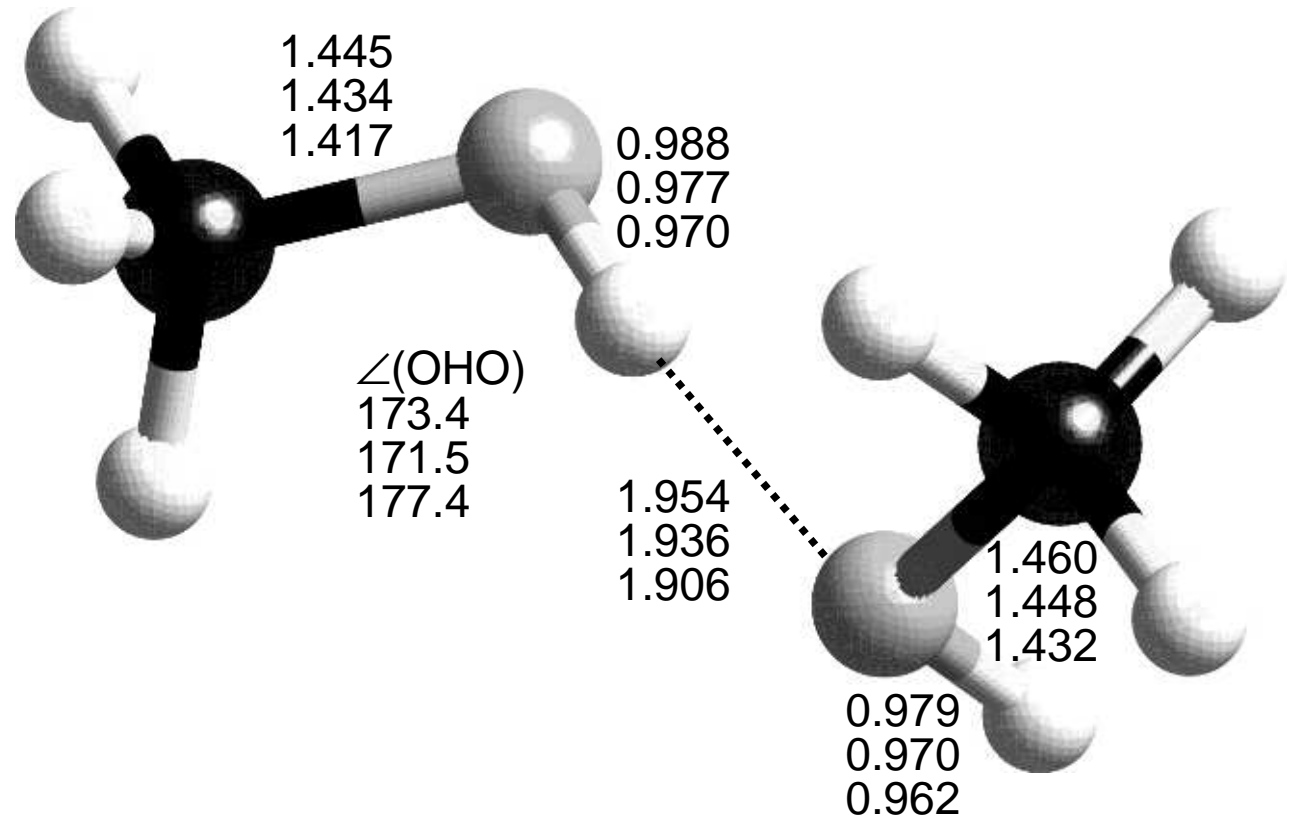

Fig. 1. Optimized geometry of the methanol dimer. Selected distances $(\AA)$ and angles (degrees) are given for three computational methods: CPMD-BLYP (top, this work), ADF-BLYP[20] (second, this work) and B3LYP[21] (third). The MP2 results of Ref. [21] are within $0.01 \AA$ of the B3LYP result. 

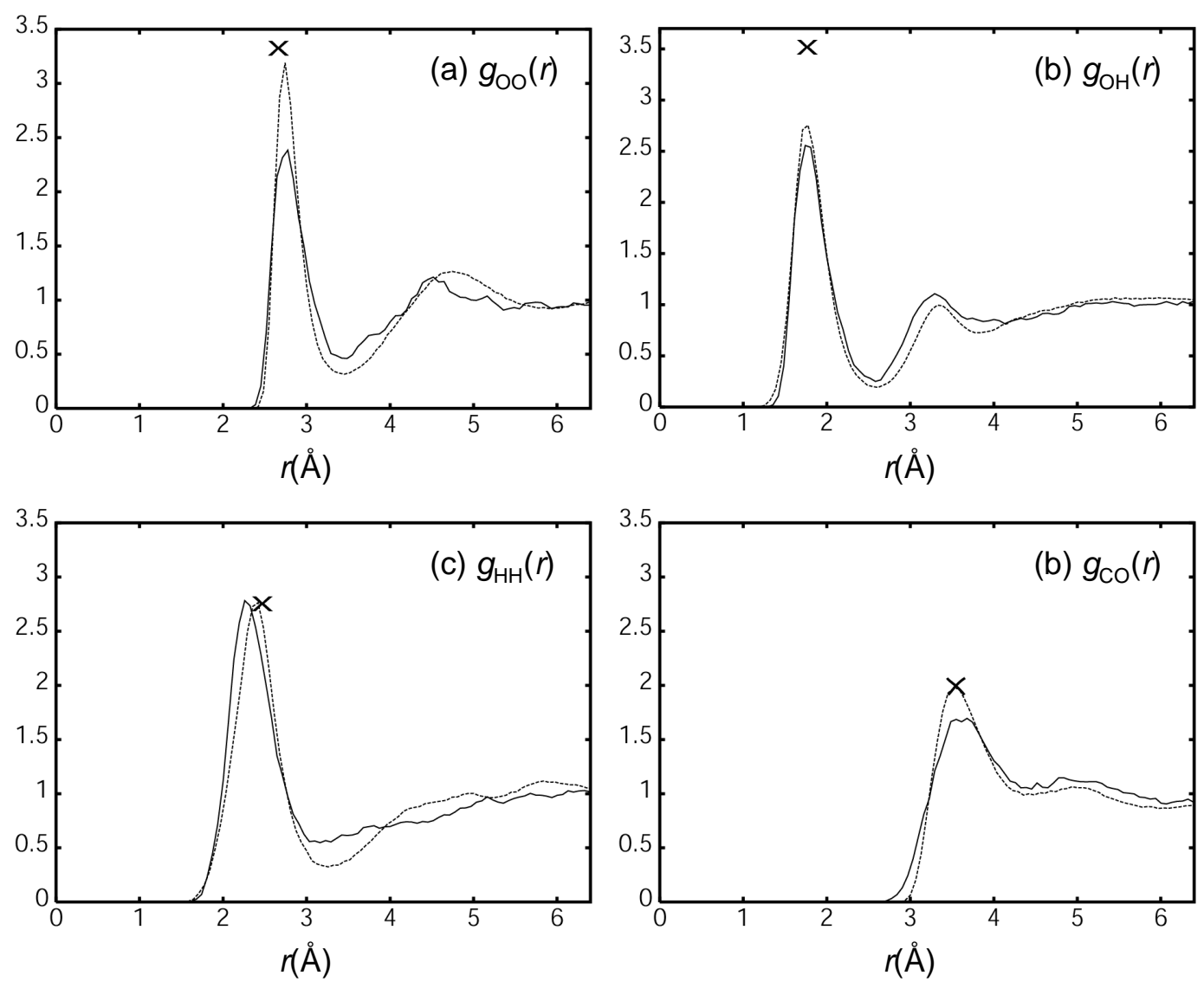

Fig. 2. Calculated hydrogen-bonding and C-O radial distribution functions (solid lines). Dashed line indicate neutron diffraction results of Ref. [1]. Crosses indicate position of the first peak of the RDFs obtained by Haugney et al. using an empirical force field[3]. 


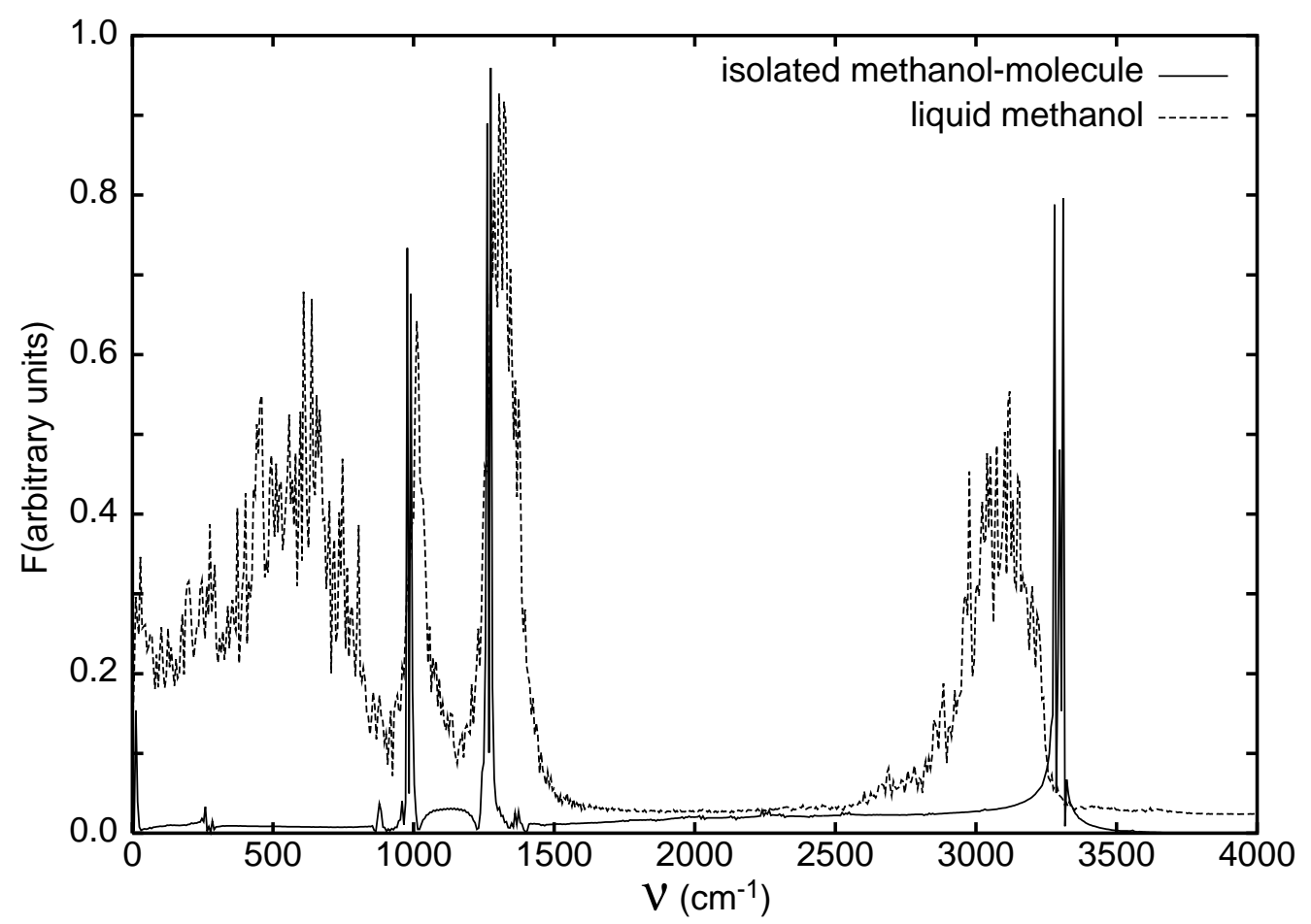

Fig. 3. Calculated power spectrum of the VACF of the hydroxyl hydrogen for an isolated methanol at $\mathrm{T}=200 \mathrm{~K}$ (solid line, from Ref. [13]) and liquid methanol at $\mathrm{T}=293 \mathrm{~K}$ (dashed line). 


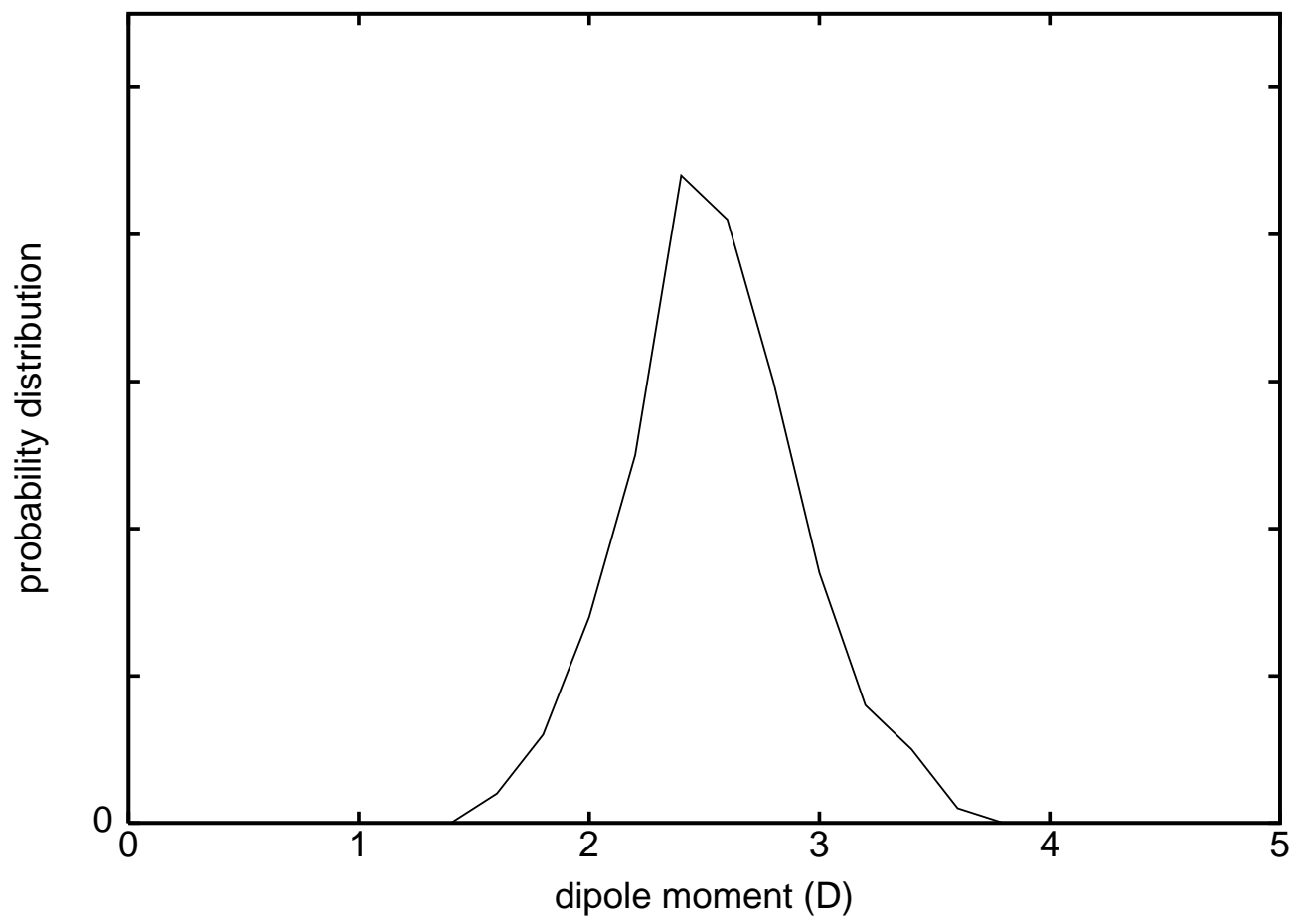

Fig. 4. Distribution of the molecular dipole moment in liquid methanol, obtained from 6 independent liquid configurations. 